\title{
EDITORIAL
}

\section{New Management Strategies of Hormone Refractory Prostate Cancer (HRPC)}

Carcinoma prostate is the commonest cancer in men and recognized as the commonest killer of men. Prostate cancer incidence is increasing in Bangladesh as the detection technology and people are servings longer. Prostate cancer progression ends up at Hormone Refractory Prostate Cancer (HRPC) or stage D3 status where no endocrine manipulation is effective. The median survival at this stage of prostate cancer is usually less than 10 months. World wide life of the most of the prostate cancer patients are terminated at this stage.

Hormone Refractory Prostate Cancer (HRPC) may occur due to the fact that prostate cancer cell escape from androgen withdrawal-induced apoptosis. In this development, enhancement of growth factor stimulation has an essential role in the up regulation of survival signals and constitutive proliferation ${ }^{1}$.

The principle of treatment for advanced prostate cancer is endocrine manipulation which includes androgen deprivation. Unfortunately, at this stage of prostate cancer most of men become resistant to hormonal manipulation, developing what is defined as hormone-refractory prostate cancer (HRPC). A decade ago, most clinicians find no answer and felt helples*ecause no Chemotherapy was considered to be ineffective and associated with unacceptable toxicity. A review of 26 chemotherapy-based trials revealed an overall response rate of $8.7 \%$ with a median survival ranging from. 6 to $10 \mathrm{mo}^{2}$. For this reason, it was established that a median expected survival for patients with HRPC is 10 months. Therefore, novel therapeutic strategies that target the molecular basis of androgen resistance were required.

Role of chemotherapy in HRPC was emphasized In 2004. Two pivotal trials of Docitaxel-based chemotherapy were reported and, for the first time, a survival benefit was observed for chemotherapy in HRPC. The results from the Southwest Oncology Group (SWOG )99-16 and TAX327 studies changed the expectations of treatment outcome these patients ${ }^{7,8}$.
Also these trials demonstrated the need for combination therapies in patients with HRPC.

The combination of Docitaxel with estramustine increases the thrombo embolic risk and necessitates a primary prophylaxis ${ }^{7,8}$. New combination models using Docitaxel may represent an exciting investigational field ${ }^{9}$. In particular, less toxic regimens, provided that the activity can be maintained, are more attractive.

Recently, Di Lorenzo et $\mathrm{al}^{9}$ presented an interesting proposal using a combination of docetaxel, vinorelbine, and zoledronic acid as first-line treatment in patients with HRPC. Vinorelbine is a vinca alkaloid that inhibits the microtubular apparatus in malignant cells and has shown activity in $\mathrm{HRPC}^{9}$. The synergism of docetaxel and vinorelbine has been confirmed in preclinical studies and human trials ${ }^{9}$. Moreover, the use of docetaxel in a weekly schedule appears to minimize myelo suppression and has been associated with moderate toxicity 9 .

Most HRPC develops bone metastases thatt are responsible for pain and morbility. Bisphosphonates showed an inhibitory effect on prostate cancer bone metastases by blocking proteolytic activity of the matrix, cell adhesion, and possibly cancer cell growth ${ }^{9}$. Multicentric randomised trials of HRPC with bone metastases showed a significant reduction in skeletal related events using zoledronic acid ${ }^{9}$.

Di Lorenzo et $\mathrm{al}^{9}$ developed a phase 2 study to evaluate the impact of weekly docetaxel and vinorelbine and monthly zoledronic acid on PSA response, pain improvement, and toxicity profile in 40 men with HRPC. Complete and partial response (PSA reduction) were observed in $18 \%$ and $32 \%$ of cases, respectively.

The objective of this editorial is to emphasizes two possible strategies: the first, specifically targeted to the role of the neuro endocrine (NE) system in 
hormone-refractory stage development, and the second, chemotherapy, not target specific and only cytotoxic.

NE activity is considered one of the factors involved in the progression from an androgendependent to an androgen-independent state and may be a possible new target therapy. In recent years a marked number of papers related to NE differentiation in prostate adenocarcinomas has published. The NE component of prostate Adenocarcinoma is androgen independent and does not produce prostate-specific antigen (PSA). The continuous use of androgen-ablation therapy may produce hyperactivation of the NE system in prostate tissue $^{3}$. NE system products can act as immortalising factors, blocking the apoptotic process in prostate adenocarcinoma cells and then inducing androgenindependent statu 5 and progression.

Several clinical trials have demonstrated impressive efficacy of somatostatin analogues for various hypersecretory disorders resistant to standard therapy. They have also proved useful for the management of symptoms caused by NE diseases. Chromogranin A $(\mathrm{CgA})$ is considered the best marker of NE activity in the prostate. In different countries $\mathrm{CgA}$ determination started to be used and to be repeated in clinical practice for the evaluation of men with prostate adenocarcinoma. The primary effect of somatostatin analogues is not a Ikect cytotoxic effect on NE cells, but rather inhibition of the release of peptide hormones secreted by NE cells. Clinical trials on somatostatin analogues as monotherapy for prostate cancer have shownknegative results ${ }^{4}$. The mechanism of action of these drugs may suggest their use not as monotherapy but rather as combination therapy for prostate cancer. Koutsilieris et $\mathrm{al}^{5}$ first proposed a combination therapy with dexamethasone and somatostatin analogues in HRPC. The author combined standard luteinising hormonereleasing hormone (LHRH) analogue therapy with somatostatin analogue and dexamethasone. Median overall survival reported in this study was $12 \mathrm{mo}$, with improvement in performance status and bone pain scores. Di Silverio and Sciarra ${ }^{6}$ analysed whether the combination of ethinyloestradiol and lanreotide can offer objective response or symptomatic improvement in patients with D3 prostate cancer. Patients with metastatic
HRPC discontinued LHRH analogue and started the combination therapy.

The rationale for this combination therapy is: (1) to inhibit the protective antiapoptotic effect of NE system on prostate adenocarcinoma cells (somatostatin analogue); (2) to use a new mechanism of castration (oestrogens); and (3) to add a direct cytotoxic effect on prostate cells (oestrogens). No major related side-effects were reported (gynaecomastia and breast pain). In this phase 2 trial, $95 \%$ of cases showed an objective clinical response as demonstrated by at least a $50 \%$ PSA decrease from baseline; in all cases the PSA response was accompanied by a significant improvement in Eastern Cooperative Oncology Group (ECOG) performance status and bone pain score; $70 \%$ of cases were without disease progression at a median of $16.5 \mathrm{mo}$ of follow-up during therapy. These results suggest the need for a phase 3 trial to confirm the effectiveness of this combination therapy in HRPC.

An objective response (liver, lung, and lymph nodes) was observed in 6 of 15 patients with measurable disease. Stratifying the response in terms of Gleason score, primary treatment, and number of osseous sites, no differences were observed among these groups. No toxic death occurred and the most important grade 3 toxicities included neutropenia $(25 \%)$. Pain improvement was found in $47.5 \%$ of cases. Median progression-free survival was $7 \mathrm{mo}$, with a median overall survival of $17 \mathrm{mo}$. The majority of patients received, after progression, a second line of chemotherapy. The rationale to improve docetaxel efficacy and to reduce the related toxicity using a combination with vinorelbine and zoledronic acid is of great interest.

(J Bangladesh Coll Phys Surg 2008; 26: 58-61)

\footnotetext{
Prof. M.A. Salam

Professor of Uro-Oncology, Department of Urology BSMMU, Dhaka.

\section{References}

1. Landstrom M, Damber JE, Bergj A. Prostatic tumor regrowth after initially successful castration therapy may be related after to a decreased apoptotic cell death rate. Cancer Res 1994;54:428195.
} 
2. Yagoda A, Petrylak D. Cytotoxic chemotherapy for advanced hormone resistant prostate cancer. Cancer

5. Sciarra A, Monti S, Gentile V, Mariotti G, Voria G, Di Silverio F. Variation in chromogranin A serum levels during intermittent versus continuous androgen deprivation therapy for prostate adenocarcinoma. Prostate 2003;55:168-79.

4. Sciarra A, Bosman C, Monti S, et al. Somatostatin analogues and estrogens in the treatment of androgen ablation refractory prostate adenocarcinoma. J Urol 2004 172:1775-83

5. Koutsilieris M, Mitsiades C, Dimopoulos T, lannidis A, Ntounis A, Lambou T. A combination therapy of dexamethasone and somatostatin analog reintroduces objective clinical response to LHRH analog in androgen ablation-refractory prostate cancer patients. J Clin Endocrinol Metab 2001;86:5729-36.

6. Di Silverio F, Sciarra A. Combination therapy of ethinyoestradiol and somatostatin analogue reintroduces objective clinical responses and decreases chromogranin A in patients with androgen ablation refractory prostate cancer. J Urol 2003;170:1812-8.

7. Tannock IF, de Wit R, Berry W. Docetaxel plus prednisone or mitoxantrone plus prednisone for advanced prostate cancer. N Engl J Med 2004;351:1502-12.

8. Petrylak DP, Tangen CM, Hussain MH. Docetaxel and estramustine compared with mitoxantrone and prednisone for advanced refractory prostate cancer. New Engl J Med 2004;351:1513-20.

9. Di Lorenzo G, Autorino R, Perdona' S, et al. Docetaxel, vinorelbine and zoledronic acid as first-line treatment in patients with hormone refractory prostate cancer: a phase II study. Eur Urol 2007;52:1020-7.

10. Saad F, Gleason DM, Murray R. Long term efficacy of zoledronic acid for the prevention of skeletal complications in patients with metastatic hormone regractory prostate cancer. J Nati Cancer Inst 2004; 96: 879-82. 